\title{
Study on Flow of Non-newtonian Power-law Fluids in Porous Media
}

\section{Xiaoguang $\mathrm{Liu}^{1, \mathrm{a}}{ }^{*}$, Xiaojun Zhou ${ }^{1, \mathrm{~b}}$, Xiaoru Hao ${ }^{1, \mathrm{c}}$, Xiaohu Sang ${ }^{1, \mathrm{~d}}$}

${ }^{1}$ School of Mechatronic Engineering and Automation, Shanghai University, Shanghai,China

axiaoguangvisa@126.com, bsdzhouxj@shu.edu.cn, ${ }^{c} x i a o r u 408 @ 126 . c o m,{ }^{d}$ sangxh7@qq.com

Keywords: power-law fluid, porous media, experimental study, numerical computation

Abstract. In this article, the porous media fluid flow experimental apparatus and five groups of solid particle parameters containing porous media are introduced. And the friction factor curve is drawn along with the change of Reynolds numbers according to the pressure drop-flow relationship. According to the flow resistance is divided into the laminar viscous resistance and the turbulent inertia resistance, the porous media flow resistance is divided into Darcy resistance and Forchheimer resistance. Compared experimental results with numerical computation, the effects of the governing parameters on the velocity profiles and pressure drops are discussed. The experimental results are close to the predicted values of friction factors from the numerical solution within a discrepancy of $15 \%$, so it is reasonable to put forward the method of volume-averaging of flow in porous media and the relevant empirical relations.

\section{Introduction}

Non-Newtonian fluid flow through porous media has been studied for a long time. Most of the earlier developments have been discussed in detail by Kemblowski and Michniewicz (1979), and Sorbie (1990). Due to the complexity of the flow phenomenon and the flow structure, the method of volume averaging of flow in porous media and averaging theorem was employed to the Navier-Stokes equations. The total drag force per unit volume was considered as the bulk damping resistance due to the porous structure and the resistance due to the inertia force, then the Brinkman-Forchheimer extended Darcy model was obtained from average momentum equation ${ }^{[1-6]}$.

In the method of volume averaging, one associates an averaging volume $V$ with every point in space, and this allows one to define an average value at every point in space. The superficial average is defined by ${ }^{[7-8]}$

$$
\langle\psi\rangle=\frac{1}{V} \int_{V_{f}} \psi d V
$$

The volume average formula of the divergence of a flow parameter $\psi$ (a spatial vector, or a second order tensor)

$$
\langle\nabla \bullet \psi\rangle=\nabla \bullet\langle\psi\rangle+\frac{1}{V} \int_{A_{s f}} n \bullet \psi d A
$$

The non-dimensional form of volume averaging of the continuity equation by

$$
\nabla^{*} \bullet U=0, U=\frac{\langle u\rangle}{u_{m}}, \nabla^{*}=D \nabla
$$

in which $U$ represents the non-dimensional superficial average velocity, $\nabla^{*}$ the non-dimensional Hamilton operator, $u_{m}$ the average velocity, $D$ the characteristic dimension, and this article adopts inner diameter of the pipe.

If the mass force is neglected, the non-dimensional form of volume averaging of transport equation is given by 


$$
\begin{aligned}
& \frac{1}{\phi^{2}} U \cdot \nabla^{*} U=-\nabla^{*} P-\frac{1}{\operatorname{ReDa}{ }^{(n+1) / 2}}|U|^{n-1} U-\frac{C_{F}^{*}}{\sqrt{D a}}|U| U+ \\
& \frac{1}{\operatorname{Re} \phi} \nabla^{*} \cdot\left[\frac{1}{\phi^{n-1}}\left|\sqrt{\frac{1}{2} \operatorname{tr}\left\langle A_{1}^{*}\right\rangle^{2}}\right|^{n-1}\left\langle A_{1}^{*}\right\rangle\right]
\end{aligned}
$$

\section{Experimental study}

This experiment adopts different concentration of polyacrylamide solutions. They have different rheological characteristics. We can obtain the pipe pressure drop in many conditions. From the relationship curve between pressure drop and Reynolds numbers, we can acquire the existence of non-Darcy flow (inertia and boundary layer influence) in porous media, and confirm the accuracy of theoretical analysis and numerical result. It has also the certain reference value in engineering seepage problems.

The experimental apparatus is comprised of water tank $\left(1 \mathrm{~m}^{3}\right)$, centrifugal pump, experimental pipe section containing porous media and control valve. The detail flow chart and instrument placement are shown in Figure 1. The experimental section is comprised of a stainless steel pipe with $0.05 \mathrm{~m}$ inner diameter and $1.5 \mathrm{~m}$ length which is full of porous media. The entire apparatus arranged 7 Keller pressure transformers (frequency is $5 \mathrm{kHz}$ ). Through the porous media fluid flow is small in the experimental, so we cannot measure it by conventional flow measurement device. For this reason, we used volumetric cylinder and seconds counter to measure fluid volume and relevant time, and took multiple average results to get the required flow.

The pressurized fluid which is provided by a centrifugal pump went through the flow control valve into porous media in the experimental section. We collected pressure signals by pressure transformer under different entrance pressures, meanwhile measured flow, and obtained flow pressure data of variation. Fig. 1 shows schematic experimental apparatus of flow in porous media.

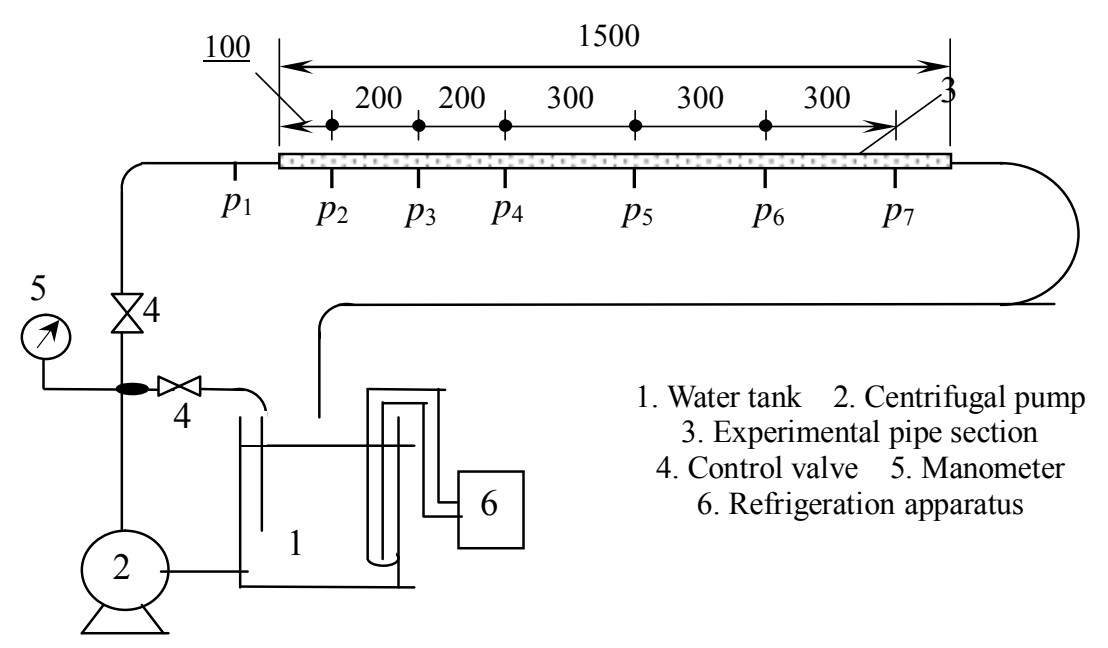

Fig. 1 Schematic experimental apparatus of flow in porous media

Five different materials respectively filled in a stainless steel pipe with $0.05 \mathrm{~m}$ inner diameter and $1.5 \mathrm{~m}$ length, and made five different porosities and permeability unconsolidated tubular porous media. Avoiding migrating outward of the particles from experimental pipe, we installed retaining sand device in experimental section of the both ends. The retaining sand device is consisted of six different diameter filters and porous circular plates. The mesh number of the filters respectively is $20,40,60,80,100$ and 140. Six measured pressure orifices in experimental section are also used the similar way to deal with it. Five kinds of materials were considered as spherical particles. The particles diameter took their weighting averaged particle size through the screening, and the particle 
density is measured by the pycnometer, water and the precision balance. The porosity of porous media is obtained by the volume measurement result, and the concrete data is shown in Table 1 .

Table 1 Parameters of porous media

\begin{tabular}{c|c|c|c|c|c|c|c}
\hline Run & Particle & $\begin{array}{c}\text { Weighting } \\
\text { averaged } \\
\text { diameter(mm) }\end{array}$ & $\begin{array}{c}\text { Coefficient of } \\
\text { shape } \\
\text { (reference[6] })\end{array}$ & $\begin{array}{c}\text { Particle } \\
\text { diameter } \\
d_{\mathrm{p}}(\mathrm{mm})\end{array}$ & $D_{\mathrm{p}}=d_{\mathrm{p}} / \mathrm{D}$ & $\begin{array}{c}\text { Density of } \\
\mathrm{particle} \rho_{\mathrm{s}} \\
\left(\mathrm{kg} / \mathrm{m}^{3}\right)\end{array}$ & Porosity $\phi$ \\
\hline 1 & Quartz sand & 1.049 & 0.591 & 0.62 & 0.0124 & 2613.18 & 0.3810 \\
\hline 2 & White grail & 2.19 & 0.63 & 1.38 & 0.0276 & 2836.45 & 0.4189 \\
\hline 3 & $\begin{array}{c}\text { Sandcontrol } \\
\text { ceramsite }\end{array}$ & 0.55 & 1.0 & 0.55 & 0.0110 & 3257.48 & 0.3998 \\
\hline 4 & Xanth grail & 2.746 & 0.63 & 1.73 & 0.0346 & 2627.02 & 0.3580 \\
\hline 5 & $\begin{array}{c}\text { Building } \\
\text { stone }\end{array}$ & 12.79 & 0.63 & 8.06 & 0.1612 & 2835.79 & 0.4393 \\
\hline
\end{tabular}

\section{Compare experimental results with numerical computational results}

The comparison of resistance factor between the experimental and computational results is shown in Fig.2. It was conducted by different polymer solution in packed bed in different experimental groups.

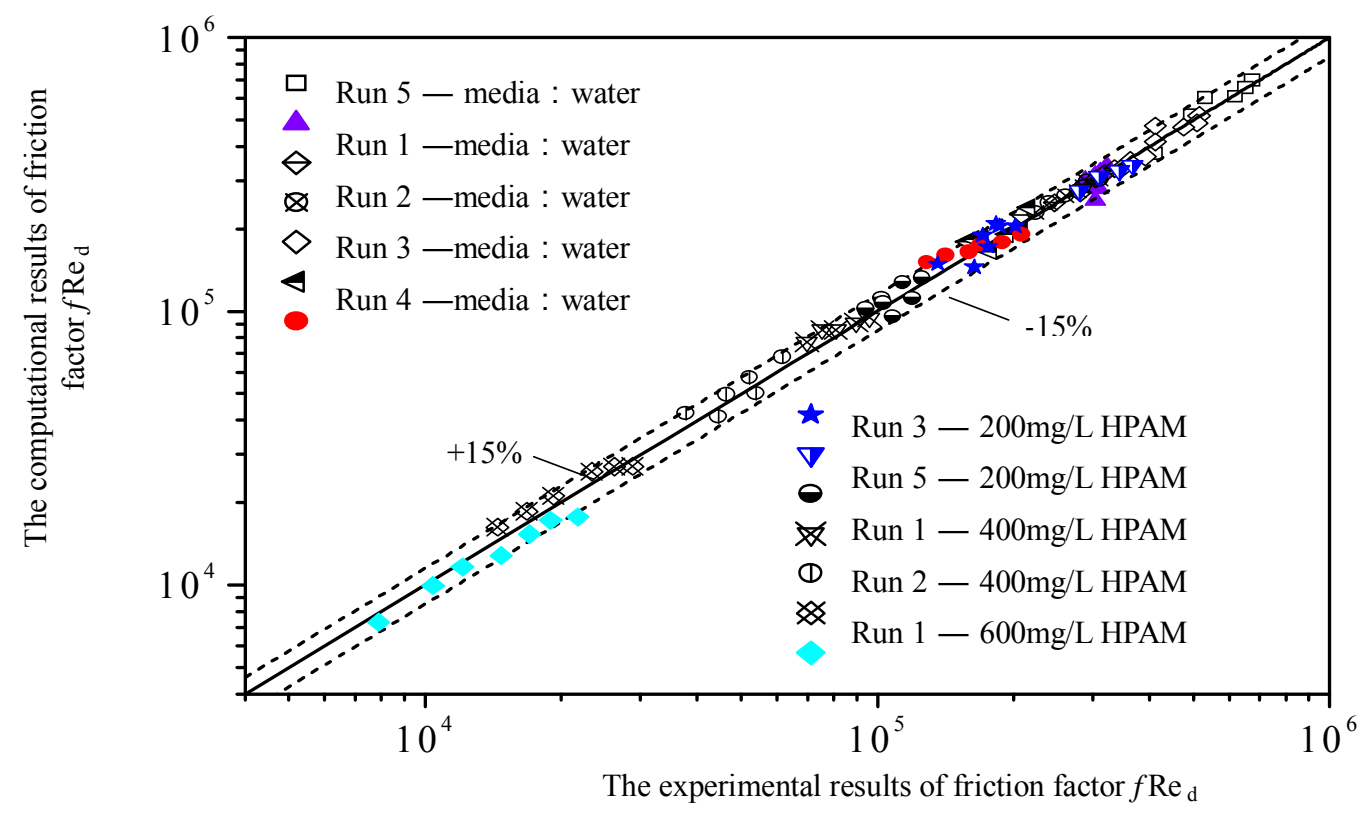

Fig. 2 Experimental results vs. computational results for flow of friction factor in packed bed

The experimental results are very close to the computational results, within $15 \%$ of the error. From this result, we can consider Brinkman-Forchheimer extended Darcy law is applied to the flow problem of uniform porous media. Forchheimer inertia resistance, Brinkman additional boundary layer viscosity resistance and the structural flow characteristics in a pipe are all confirmed indirectly. In this article, we use the exponential form to Darcy resistance of power-law fluid flow in porous media, and it also has a better application in numerical computation. Comparing to the experimental result, it verify the efficiency of the point. 


\section{Conclusions}

(1) This article introduced the porous media fluid flow experimental apparatus and five groups of solid particle parameters. Through the pressure drop-flow relationship, we draw the resistance factor curve along with the change of Reynolds numbers.

(2) The flow resistance of the numerical results agree well with the experiment, is illustrated in this article the method of volume averaging and related empirical formula is effective.

\section{References}

[1] WANG Ke-liang, LIANG Shou-cheng and YUAN Xin-qiang. Seepage ability of high-pressure hot composite foam in porous media, Journal of Hydrodynamics, 2010,22(1):90-95

[2] VINAY G., WACHS A. and AGASSANT J.F. Numerical simulation of weakly compressible Bingham flows: The restart of pipeline flows of waxy crude oils, Journal of Non-Newtonian Fluid Mechanics, 2006,136(2-3):93-105

[3] Inoue M, Nakayama A. Numeri cal modeling of non-Newtonian fluid flow in a porous medium using a three-dimensional periodic array,Journal of Fluids Engineering , 1998, 120: $131 \sim 135$.

[4] Prasad V. Thermal convection in a rectangular cavity filled with a heat-generating, Darcy porous media. Journal of Heat Transfer , 1987, 109: $697 \sim 703$.

[5] Lauriat G, Prasad V. Natural convection in a vertical porous cavity: a numerical study for Brinkman -extended Darcy formulation. Journal of Heat Transfer, 1987, 109: $688 \sim 696$.

[6] VALDES-PARADA F.J.,OCHOA-TAPIA J. A. and ALVAREZ-RAMIREZ J. Validity of the permeability Carman-Kozeny equation: A volume averaging approach, Physica A: Statistical Mechanics and its Applications,2009,388(6):789-798.

[7] Amna Gumati, Hiroshi Takahshi. Experimental study and modeling of pressure loss for foam-cuttings mixture flow in horizontal pipe,Journal of Hydrodynamics, 2011, 23(4):431-438

[8] ROOS M.,BATAWI E. And HARNISCH U. et al. Efficient simulation of fuel cell stacks with the volume averaging method, Journal of Power sources, 2003,118(1-2):86-95. 